\title{
2,5-二取代噻吩衍生物的“一锅化”串联合成及光学性质
}

\author{
钱存卫* 媙世宇周倩王栋李万金金茂元* \\ (盐城师范学院 化学与环境工程学院 盐城 224051)
}

\begin{abstract}
摘要 以芳基乙炔与硫脲为起始原料, 通过一锅法, 经过在无溶剂条件下的氧化偶联反应和在水相中的成环反应, 两 步串联绿色合成了 2,5 -二苯基噻吩及其衍生物. 所合成化合物经 ${ }^{1} \mathrm{H} N M R,{ }^{13} \mathrm{C} N M R, H R M S$ 表征, 均为目标产物. 在此 基础上，进一步探索了所合成化合物的 UV 和苂光性质，实验结果表明，所合成化合物的紫外最大吸收波长在 292 $341 \mathrm{~nm}$ 之间，苂光光谱表明该类化合物具有良好的苂光性，在甲醇中最大发射波长在 386 454.5 nm之间，在二氯甲烷 中测定的最大发射波长在 390 412 nm 之间. 实验结果显示，随共轭体系增大，苂光发射波长发生红移.

关键词 芳基乙炔; 硫尿; 噻吩衍生物; “一锅化” 合成; 光学性质
\end{abstract}

\section{One-Pot Synthesis and Optical Properties of 2,5-Diphenylthiophene Derivatives}

\author{
Qian, Cunwei* Zang, Shiyu Zhou, Qian $\quad$ Wang, Dong
}

Li, Wanxin Wang, Maoyuan*

(Yancheng Teachers College, School of Chemical \& Environmental Engineering, Yancheng 224051)

\begin{abstract}
The paper describes a convenient and facile methodology for the synthesis of 2,5-diphenylthiophene derivatives. The environmentally friendly synthetic approach is supported by a one-pot tandem reaction process. All of the target products was confirmed by ${ }^{1} \mathrm{H}$ NMR, ${ }^{13} \mathrm{C}$ NMR and HRMS. On this basis, UV and fluorescence properties of the synthesized compounds were further explored. The experimental results showed that the UV maximum absorption wavelengths of the compounds are between 292 and $341 \mathrm{~nm}$. The fluorescence spectra showed that these compounds have good fluorescence. The fluorescence emission wavelengths measured in methanol are between 386 and $454.5 \mathrm{~nm}$, and the fluorescence emission wavelengths measured in dichloromethane are between 390 and $412 \mathrm{~nm}$. The increase of conjugation system led to the red shift of fluorescence.
\end{abstract}

Keywords aryl acetylene; urinary sulfur; thiophene derivative; one-pot synthesis; optical properties

噻吩是一个基本五元芳香杂环, 含噻吩结构的天然 产物大多具有良好的生物活性, 而且噻吩衍生物的聚合 物在现代光电子、微电子领域及现代医学等领域中也具 有诱人的应用前景, 因而新噻吩衍生物和新合成方法的 研究一直受到关注 ${ }^{[1]}$. 噻吩及其衍生物的经典制备方法 是 Paal-Knorr 反应, Paal-Knorr 反应是利用 1, 4-二羰基化 合物与含硫化合物缩合生成噻吩及其衍生物 ${ }^{[2]}$. 此外, 化学工作者也在探索以其它化合物为原料的噻吩及其 衍生物的合成方法 ${ }^{[3]}$, 经探索发现 1,3-丁二炔衍生物作 为一种重要的合成中间体, 可以用于各种环状化合物的 合成 ${ }^{[4]}$, 而且使用 1,3 -丁二炔及其衍生物作为原料时,
反应具有原子经济性、原料简单易得等特点. 因而，近 来有一批文献报道了一些以 1,3-丁二炔衍生物为原料的 合成噻吩及其衍生物的新方法 ${ }^{[5]}$. 例如 Hua 等 $^{[5 \mathrm{a}]}$ 报道了 钯催化或者直接采用氢氧化钾促进炔烃与 $\mathrm{Na}_{2} \mathrm{~S}$ 反应, 高产率地合成对称的 2,5-二取代噻吩的方法. Zhang 等 ${ }^{[5 b]}$ 报道了碱促进的中间体 $\mathrm{S}_{3}$ 自由基参与的 1,3-丁二 炔及其衍生物环化生成 2,5-二取代噻吩的合成方法, 该 方法操作简单, 产物易分离. Zhao 等 ${ }^{[5 c]}$ 报道了 NaHS 可 以在无金属催化剂存在的条件下与 1,3 -丁二炔反应高产 率地生成 2,5-二取代噻吩. 尽管利用上述方法已经可以 有效合成噻吩类化合物，但鉴于噻吩化合物在实际应用

* Corresponding author. E-mail: qiancunwei@163.com

Received December 15, 2016; revised February 22, 2017; published online March 8, 2017.

Project supported by the Natural Science Foundation of the Jiangsu Higher Education Institutions (No. 14JKD150009) and the Excellent Specialties Program Development of Jiangsu Higher Education Institutions.

江苏省高校自然科学基金(No. 14JKD150009)、江苏高校品牌专业建设工程资助项目. 
中的重要性, 我们认为发展从简单易得原料出发, 更加 简单高效、绿色合成取代噻吩杂环的合成方法仍然很有 必要. 因此, 本课题组将报道一个绿色的, 高效地合成 2,5-二取代噻吩的新方法(Scheme 1), 并将对合成出噻 吩衍生物进行初步的光学性质研究.

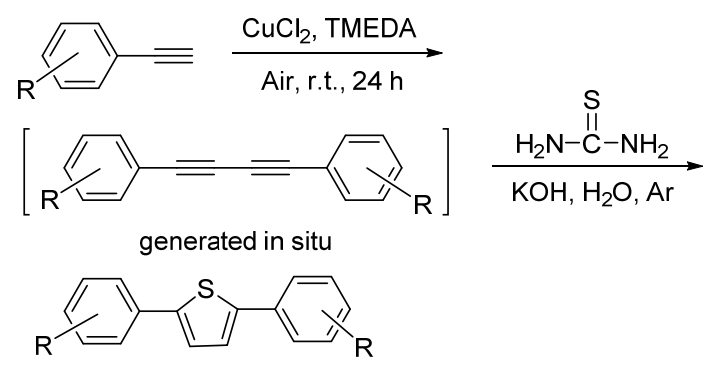

图式 12,5 -二芳基噻吩的合成设计

Scheme 1 Design for syntheses of 2,5-diarylthiophene

\section{1 结果与讨论}

\section{1 最佳反应条件的建立}

我们采用苯乙炔作为反应原料经过偶联、环化等反 应串联合成了 2,5 -二苯基噻吩. 实验过程中首先以空气 为氧化剂, 以铜化合物为催化剂, 在室温无溶剂的条件 下, 苯乙炔经过 $24 \mathrm{~h}$ 氧化偶联得到 1,3-二炔类化合物; 然后不经分离, 在反应混合物中加入水为溶剂, 以 TEBA 为相转移催化剂, 以硫代有机物、 NaHS 为硫源, 在 $\mathrm{Ar}$ 保护碱性条件下, 于 $120{ }^{\circ} \mathrm{C}$ 经过一定时间的反应, 环化生成 2,5-二取代噻吩化合物. 探索了合成 2,5-二取 代噻吩的反应条件. 实验结果见表 1.

从表 1 中的数据可以看出, 二氯化铜的催化效果优 于其它铜盐(表 1, Entries 1 5). 随后, 我们以二氯化铜 为催化剂, 考察了各种配体对反应的影响. 实验结果显 示以 TMEDA 为辅助配体时, 反应收率最高, 达 $71.0 \%$ (表 1, Entries 1, 6 9). 接着, 考察了各种碱对反应的影 响. 在被考察的碱中, $\mathrm{KOH}$ 得到了最高的收率, 而其它 碱虽有一定效果, 但与 $\mathrm{KOH}$ 相比, 结果都不太理想(表 1, Entries 1, 10 12), 因此, 在后续反应中, 我们均以 $\mathrm{KOH}$ 作为碱来考察其它因素对反应的影响. 考察不同 硫源对反应的影响结果显示, 相对于硫代乙酰胺, 硫尿 作为硫源时，反应收率更高，达到 76.1\%(表 1, Entry 13); 而使用 $\mathrm{NaHS}$ 作为硫源时, 反应收率只有 $45 \%$ (表 1 , Entry 14). 最后考察了时间对该反应的影响, 尝试延长 环化反应时间到 36 h, 产率升高到 89.3\%(表 1, Entry 15).

\subsection{2,5-二苯基噻吩衍生物的合成}

在得到了合成 2,5-二苯基噻吩的最佳反应条件之 后, 我们进一步探索了不同芳基端炔在该反应条件下的
表 1 反应条件优化 ${ }^{a}$

Table 1 Optimization of reaction conditions

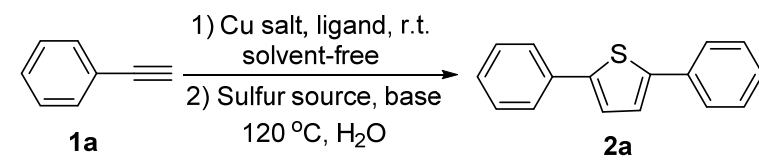

\begin{tabular}{cclccc}
\hline Entry & Catalyst & \multicolumn{1}{c}{ Ligand } & Base & $\begin{array}{c}\text { Reaction } \\
\text { time/h }\end{array}$ & Yield $/ \%$ \\
\hline 1 & $\mathrm{CuCl}_{2} /$ & TMEDA & $\mathrm{KOH}$ & 24,24 & 71.0 \\
2 & $\mathrm{CuI}$ & TMEDA & $\mathrm{KOH}$ & 24,24 & 46.6 \\
3 & $\mathrm{CuCl}$ & TMEDA & $\mathrm{KOH}$ & 24,24 & 29.0 \\
4 & $\mathrm{Cu}_{2} \mathrm{O}$ & TMEDA & $\mathrm{KOH}$ & 24,24 & 22.4 \\
5 & $\left.\mathrm{Cu}^{\mathrm{acac}}\right)_{2}$ TMEDA & $\mathrm{KOH}$ & 24,24 & 13.8 \\
6 & $\mathrm{CuCl}_{2}$ & TEA & $\mathrm{KOH}$ & 24,24 & 53.9 \\
7 & $\mathrm{CuCl}_{2}$ & Oxine & $\mathrm{KOH}$ & 24,24 & 48.4 \\
8 & $\mathrm{CuCl}_{2}$ & $1,10-P h e n a n t h r o l i n e$ & $\mathrm{KOH}$ & 24,24 & 46.2 \\
9 & $\mathrm{CuCl}_{2}$ & Methenamine & $\mathrm{KOH}$ & 24,24 & 45.4 \\
10 & $\mathrm{CuCl}_{2}$ & TMEDA & $\mathrm{NaOH}$ & 24,24 & 49.9 \\
11 & $\mathrm{CuCl}_{2}$ & TMEDA & $\mathrm{NaOAc}$ & 24,24 & 34.0 \\
12 & $\mathrm{CuCl}_{2}$ & TMEDA & $\mathrm{K} 2 \mathrm{CO}$ & 24,24 & 47.7 \\
$13^{c}$ & $\mathrm{CuCl}_{2}$ & TMEDA & $\mathrm{KOH}$ & 24,24 & 76.1 \\
$14^{d}$ & $\mathrm{CuCl}_{2}$ & TMEDA & $\mathrm{KOH}$ & 24,24 & 45.0 \\
$15^{c}$ & $\mathrm{CuCl}_{2}$ & TMEDA & $\mathrm{KOH}$ & 24,36 & 89.3 \\
\hline
\end{tabular}

${ }^{a}$ General reaction conditions: phenylacetylene $(2 \mathrm{mmol}), \mathrm{Cu}$ compounds $(1$ mol\%), ligand ( $5 \mathrm{~mol} \%)$, room temperature; base ( $2.0 \mathrm{mmol}$, cyclic reaction), $\mathrm{H}_{2} \mathrm{O}$ (1.0 mL, cyclic reaction), TAA (1.5 mmol, cyclic reaction), under $\mathrm{Ar}$ (cyclic reaction), TEBA (5 mol\%, cyclic reaction), $120{ }^{\circ} \mathrm{C}$ (cyclic reaction). ${ }^{b}$ Isolated yield. ${ }^{c} 1.5 \mathrm{mmol}$ of thiourea was used in place of TAA. ${ }^{d} 1.5 \mathrm{mmol}$ of NaHS was used in place of TAA.

反应活性，实验结果见表 2 .

从表 2 中的数据可以看出, 在该反应条件下, 以各 种芳基端炔为原料的反应均可以高效地合成 2,5-二取代 噻吩, 实验结果也显示, 当苯环对位上有强供电子基团 时, 反应的收率有所降低(表 2, Entries 3,5); 当苯环的 邻、对位上有弱供电子基时, 反应的收率变化不大(表 2, Entries 1, 2, 4, 7); 当苯环被吡啶环取代时, 反应的收 率变化也不大(表 2, Entry 8). 实验结果还显示苯环间位 上的氨基对反应也没有明显的影响(表 2, Entry 6).

\section{3 可能的反应机理}

根据文献 $[5 b, 6]$, 我们认为该合成过程是由两步串 联而成, 第一步是端炔在铜催化下氧化偶联生成 1,3-丁 二炔，第二步是硫尿在碱作用下形成 $\mathrm{S}^{-}$离子, $\mathrm{S}^{-}$离子通 过两次加成-环化生成 2,5-二取代噻吩. 具体的反应机 理见 Scheme 2.

\section{$1.42,5$-二苯基噻吩类化合物的光学性质}

\subsubsection{2,5-二苯基噻吩类化合物的紫外光谱}

从上述合成出的 8 种 2,5 -二取代噻吩化合物中选择 化合物 $\mathbf{2 a}, \mathbf{2} \mathbf{e} \sim \mathbf{2 i}$ 为代表, 研究了 2,5 -二取代噻吩化合 物的紫外光谱. 图 1 为所选择的化合物在 $\mathrm{CH}_{3} \mathrm{OH}(1 \times$ 
表 2 2,5-二苯基噻吩类化合物的合成 ${ }^{a}$

Table 2 Synthesis of 2,5-phenylthiophene derivatives

$$
\mathrm{Ar} \equiv \frac{\text { (1) } \mathrm{CuCl}_{2}, \mathrm{TMEDA}, \text { Air, r.t., } 15 \mathrm{~h}}{\text { (2) Thiourea, } \mathrm{KOH}, \mathrm{H}_{2} \mathrm{O}, \mathrm{Ar}, 120^{\circ} \mathrm{C}, 36 \mathrm{~h}}
$$

Entry

${ }^{a}$ General reaction conditions: Alkyne $(2 \mathrm{mmol}), \mathrm{CuCl}_{2}(1 \mathrm{mmol} \%)$, TMEDA $(5 \mathrm{mmol} \%)$, at room temperature; $\mathrm{KOH}\left(2 \mathrm{mmol}, \mathrm{Cyclic}\right.$ reaction), $\mathrm{H}_{2} \mathrm{O}(1.0 \mathrm{~mL}$, Cyclic reaction), Thiourea (1.5mmol, Cyclic reaction), Under Ar (Cyclic reaction), TEBA (5mmol\%, Cyclic reaction), At $120{ }^{\circ} \mathrm{C}$ (Cyclic reaction). ${ }^{b}$ Isolated yield.

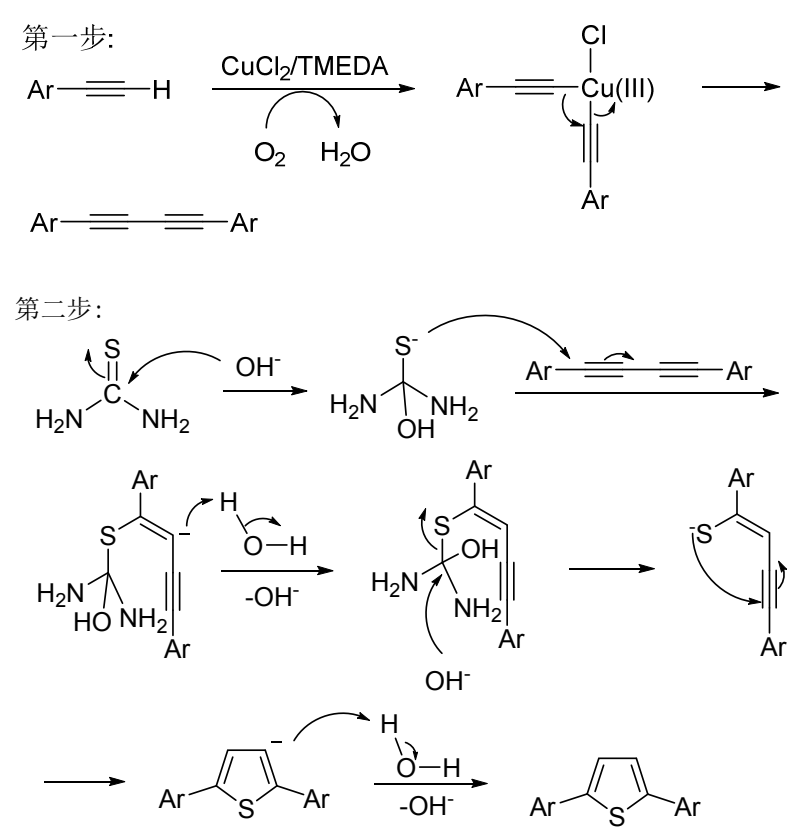

图式 2 可能的反应机理

Scheme 2 Possible reaction mechanism

$\left.10^{-5} \mathrm{~mol} / \mathrm{L}\right)$ 中的紫外-可见吸收光谱(UV-Vis).

如图 1 所示, 所选择的化合物 $2 \mathbf{a}, 2 \mathrm{e}, 2 \mathbf{f}, 2 \mathrm{~g}, 2 \mathbf{h}$ 和 2i 的紫外最大吸收波长位于 292 341 nm 之间, 这一范 围的吸收是由共轭体系中噻吩环和芳环共轭的 $\pi-\pi *$ 跃 迁引起的, 由于芳环的不同, 或由于苯环上取代基的不 同，导致了所选择的化合物 $\mathbf{2 a}, \mathbf{2} \mathrm{e} \sim \mathbf{2 i}$ 的紫外最大吸收

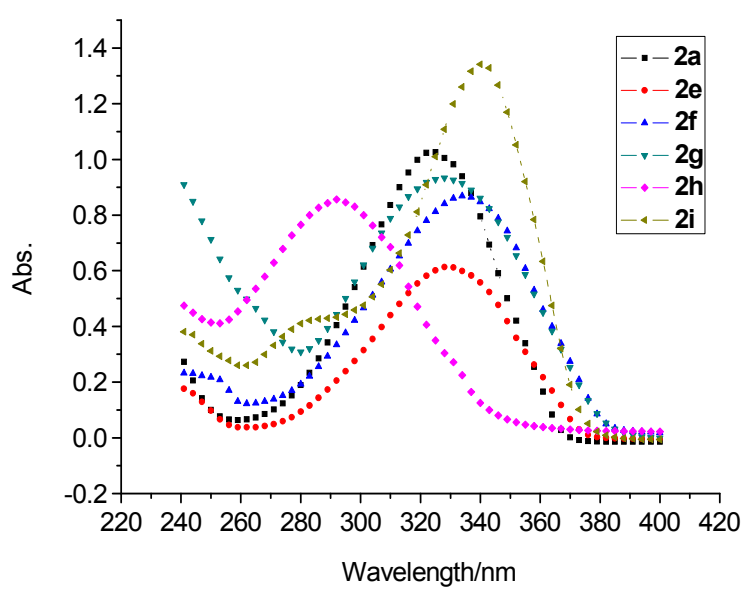

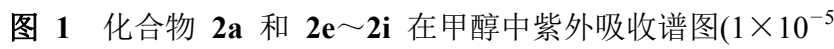
$\mathrm{mol} / \mathrm{L}$ )

Figure 1 UV-Vis spectra of compounds $\mathbf{2 a}$ and $\mathbf{2 e} \sim \mathbf{2} \mathbf{i}$ in methanol $\left(1 \times 10^{-5} \mathrm{~mol} / \mathrm{L}\right)$

波长在 292 341 nm 之间波动. $2 \mathbf{a}$ 的最大紫外吸收波长 为 $324 \mathrm{~nm}, \mathbf{2 e}$ 相对 $\mathbf{2 a}$ 来说，苯基的 4-位上有甲基取代 基，甲基与苯环上的 $\pi_{6}^{6}$ 键存在着超共轭效应, $2 \mathrm{e}$ 的最大 紫外吸收波长小幅向长波方向移动, 为 $329 \mathrm{~nm}$. $2 \mathrm{f}$ 相对 于 $\mathbf{2} \mathbf{a}$ 来说，苯基的 4-位上有甲氧基取代基，甲氧基中的 氧上的 $\mathrm{p}$ 电子对与苯环上的 $\pi_{6}^{6}$ 键存在着共轭效应, $2 \mathbf{f}$ 的 最大紫外吸收波长向长波方向移动, 为 $334 \mathrm{~nm} . \mathbf{2 g}$ 相对 于 $2 \mathbf{a}$ 来说，苯基的 3-位上有氨基取代基，氨基中的氮上 的 $\mathrm{p}$ 电子对与苯环上的 $\pi_{6}^{6}$ 键存在着共轭效应, $2 \mathrm{~g}$ 的最大 
紫外吸收波长向长波方向移动, 为 $329 \mathrm{~nm}$. $2 \mathbf{h}$ 相对 $\mathbf{2 a}$ 来说, 苯基的 2-位上有甲基取代基, 由于甲基的体积位 阻较大, 导致了苯环与噻吩环不能很好地共平面, 破坏 了苯环与噻吩环的共轭效应, $2 \mathrm{e}$ 的最大紫外吸收波长向 短波方向移动, 为 $292 \mathrm{~nm}$. 2i 相对于 $\mathbf{2 a}$ 来说, 苯环被置 换成了吡啶环, $2 \mathbf{i}$ 的最大紫外吸收波长为 $341 \mathrm{~nm}$.

\subsubsection{2,5-二苯基噻吩类化合物的荧光光谱}

用甲醇、二氯甲烷分别作溶剂将 $2 \mathbf{a}, 2 \mathrm{e} \sim 2 \mathbf{i}$ 配成 $5 \times 10^{-6} \mathrm{~mol} / \mathrm{L}$ 的浓度, 测得紫外吸收光谱, 分别得到相 应的最大吸收波长和它对应的吸光度值 $A$, 再用最大吸 收波长作为激发波长, 测定它们的发射光谱 $\lambda_{\mathrm{EM} 1}$, 再以 $\lambda_{\mathrm{EM} 1}$ 最大值测定激发光谱 $\lambda_{\mathrm{EX}}$, 最后再以 $\lambda_{\mathrm{EX}}$ 最大值测定 目标产物的荧光发射光谱 $\lambda_{\mathrm{EM} 2}$, 测定结果如图 2 和图 3 所示. 图 2 为上述化合物在甲醇中的苂光发射光谱, 从 图 2 中可以看出, 上述化合物的最大发射波长在 386 $454.5 \mathrm{~nm}$ 之间, 化合物 $2 \mathrm{e}, \mathbf{2 f}, \mathbf{2 g}$ 和 $\mathbf{2 i}$ 的最大荧光发射 波长相比 $2 \mathrm{a}$ 的最大发射波长发生了红移, 尤其是 $2 \mathrm{~g}$ 的 红移(67 nm) 最为明显, 这可能是取代基使共轭体系增 大的缘故; $\mathbf{2 h}$ 的最大荧光发射波长相比 $\mathbf{2 a}$ 的最大发射 波长, 发生了蓝移, 这可能是由于甲基在苯环的 2 位时, 位阻破坏了苯环与噻吩环的有效共轭. 图 3 为上述化合 物在二氯甲烷中的苂光发射光谱, 从图 3 中可以看出, 上述化合物的最大发射波长在 $390 \sim 412 \mathrm{~nm}$ 之间, 化合 物 $2 \mathrm{e}, 2 \mathrm{f}, 2 \mathrm{~g}$ 和 $2 \mathrm{i}$ 的最大荧光发射波长相比 $2 \mathrm{a}$ 的最大发 射波长发生了红移, 这可能是取代基使共轭体系增大的 缘故; $\mathbf{2 h}$ 的最大苂光发射波长相比 $\mathbf{2 a}$ 的最大发射波长 发生了蓝移, 这可能是由于 2 位上的甲基的位阻使苯环 与噻吩环不能有效共轭. 从实验结果可以发现, 上述化 合物在甲醇中苂光强度要强一些, 当苯环 3 位上取代基 是氨基时, 化合物的最大荧光发射波长在甲醇中的红移 明显, 在二氯甲烷中只有轻微的红移.

\section{2 结论}

本文以芳基乙炔与硫腿为起始原料, 通过一锅法, 经过氧化偶联反应和成环反应两步串联, 合成了 $2,5-$ 二 苯基噻吩及其衍生物. 在此基础上, 进一步探索了所合 成化合物的 UV 和苂光性质, 实验结果表明所合成化合 物在甲醇中的紫外最大吸收波长在 292 341 nm 之间, 荧光光谱表明该类化合物具有良好的荧光性，其在甲醇 中最大发射波长在 386 454.5 nm 之间，在二氯甲烷中 测定的最大发射波长在 390 412 nm 之间.

\section{3 实验部分}

\section{1 仪器与试剂}

高分辨质谱测试: AB SCIEX Triple 5600+型液质

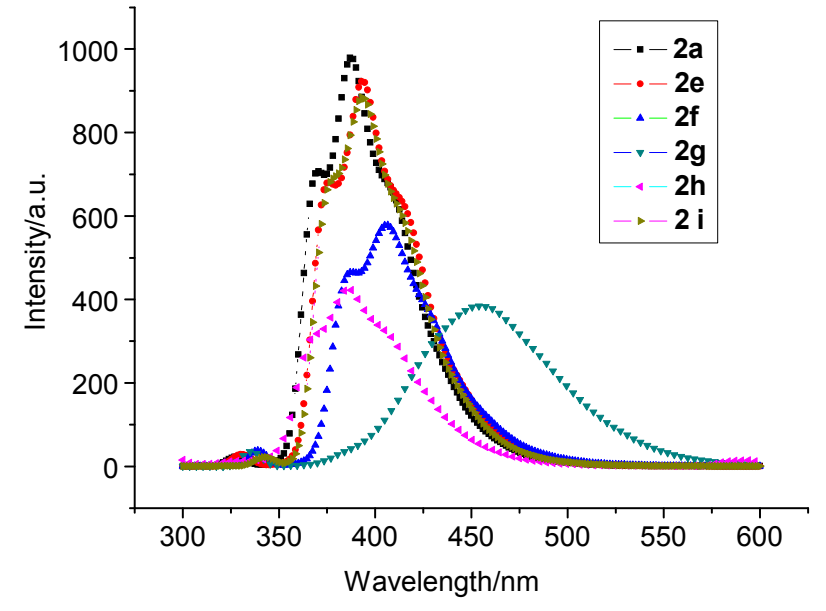

图 2 化合物 $2 \mathrm{a}$ 和 $\mathbf{2 e} \sim \mathbf{2 i}$ 在甲醇中的苂光光谱 $\left(5 \times 10^{-6}\right.$ $\mathrm{mol} / \mathrm{L}$ )

Figure 2 Fluorescence spectra of compounds $\mathbf{2 a}$ and $\mathbf{2 e} \sim \mathbf{2 i}$ in methanol $\left(5 \times 10^{-6} \mathrm{~mol} / \mathrm{L}\right)$

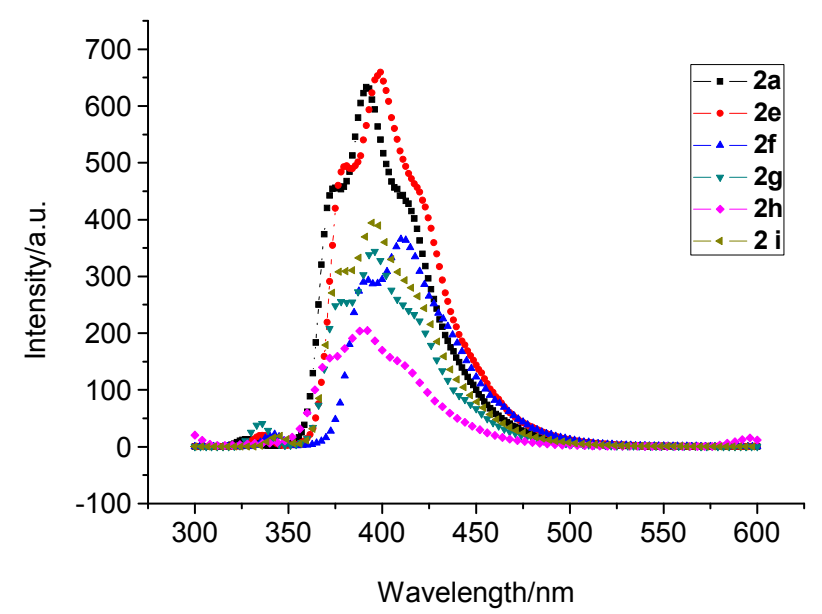

图 3 化合物 $\mathbf{2 a}$ 和 $\mathbf{2 e} \sim \mathbf{2 i}$ 在二氯甲烷中的苂光光谱 $\left(5 \times 10^{-6}\right.$ $\mathrm{mol} / \mathrm{L}$ )

Figure 3 Fluorescence spectra of compounds $\mathbf{2 a}$ and $\mathbf{2 e} \sim \mathbf{2} \mathbf{i}$ in dichloromethane $\left(5 \times 10^{-6} \mathrm{~mol} / \mathrm{L}\right)$

连用仪(美国 AB SCIEX 公司). ${ }^{1} \mathrm{H}$ NMR 和 ${ }^{13} \mathrm{H}$ NMR 谱: Mercury Plus 400 型核磁共振仪(美国 Varian 公司), $\mathrm{CDCl}_{3}$ 为溶剂, TMS 为内标. 紫外吸收光谱: Lambda 950 紫外可见分光光度计上测定, 测试溶液样品使用的样品 池是通光长度为 $1.0 \mathrm{~cm}$ 的两面通光的石英池. 苂光光谱 测试：带恒温系统的 LS-50B 型荧光光度计 (美国 Perkin-Elmer 公司) 测定, 测试溶液样品使用的样品池为 $1.0 \mathrm{~cm} \times 1.0 \mathrm{~cm}$ 四面通光的石英比色典.

所用的化学原料试剂全部从 Alfa、Aldrich、百灵威 等公司购买，没有进一步的纯化; 常用溶剂购自国药集 团; 薄层层析硅胶由硅胶 GF254 (青岛海洋化工厂) 和羧 甲基纤维素 $(\mathrm{CMC})$ 自制; 柱层析用硅胶为化学纯试剂 (青岛海洋化工厂). 


\section{2 实验方法}

\subsubsection{2,5-二取代噻吩的合成}

往反应管中依次加入搅拌磁子、末端炔 $(2 \mathrm{mmol})$ 、 $\mathrm{CuCl}_{2}$ (5 mol $\%$ )和四甲基乙二胺(TMEDA) ( $5 \mathrm{~mol} \%$ ), 反 应在室温摚拌反应 $24 \mathrm{~h}$; 然后加入 $2 \mathrm{~mL}$ 纯水、硫尿 $(1.5$ mmol), TEBA (5 mmol $\%)$ 和 $\mathrm{KOH}(2 \mathrm{mmol})$, 升温至 $120{ }^{\circ} \mathrm{C}$ 下摚拌反应 $36 \mathrm{~h}$ 至薄层色谱监测原料完全转化, 直接中性氧化铝拌样经柱层析纯化, 使用 $V$ (石油醚)： $V($ 二氯甲烷 $)=15: 1$ 作为淋洗剂, 得到产物 2 .

2,5-二苯基噻吩 $(\mathbf{2 a})^{[7]}: 211 \mathrm{mg}$, 白色固体, m.p. $150 \sim 152{ }^{\circ} \mathrm{C}$ (lit. $\left.{ }^{[7]} 153{ }^{\circ} \mathrm{C}\right) ;{ }^{1} \mathrm{H} \mathrm{NMR}\left(\mathrm{CDCl}_{3}, 400 \mathrm{MHz}\right)$ $\delta: 7.26 \sim 7.30(\mathrm{~m}, 4 \mathrm{H}, \mathrm{ArH}), 7.39(\mathrm{t}, J=8.0 \mathrm{~Hz}, 4 \mathrm{H}, \mathrm{PhH})$, $7.64(\mathrm{~d}, J=7.6 \mathrm{~Hz}, 4 \mathrm{H}, \mathrm{PhH}) ;{ }^{13} \mathrm{C} \mathrm{NMR}\left(\mathrm{CDCl}_{3}, 100\right.$ MHz) $\delta$ : 143.6, 134.3, 128.9, 127.5, 125.7, 124.0; HRMS (ESI) calcd for $\mathrm{C}_{16} \mathrm{H}_{13} \mathrm{~S}[\mathrm{M}+\mathrm{H}]^{+}$237.0738, found 237.0733 .

2,5-二(4'-乙基苯基)噻吩 (2b): $211 \mathrm{mg}$, 淡黄色固体, m.p. $170 \sim 172{ }^{\circ} \mathrm{C} ;{ }^{1} \mathrm{H}$ NMR $\left(\mathrm{CDCl}_{3}, 400 \mathrm{MHz}\right) \delta: 1.25$ (t, $\left.J=7.6 \mathrm{~Hz}, 6 \mathrm{H}, \mathrm{CH}_{3}\right), 2.66$ (q, $\left.J=7.6 \mathrm{~Hz}, 4 \mathrm{H}, \mathrm{CH}_{2}\right), 7.20$ (s, 2H, ArH), 7.23 (t, $J=7.6 \mathrm{~Hz}, 4 \mathrm{H}, \mathrm{PhH}), 7.54$ (d, $J=8.0$ $\mathrm{Hz}, 4 \mathrm{H}, \mathrm{PhH}) ;{ }^{13} \mathrm{C}$ NMR $\left(\mathrm{CDCl}_{3}, 100 \mathrm{MHz}\right) \delta: 143.7$, 143.3, 131.9, 128.4, 125.6, 123.5, 28.6, 15.6; HRMS (ESI) calcd for $\mathrm{C}_{20} \mathrm{H}_{21} \mathrm{~S}[\mathrm{M}+\mathrm{H}]^{+}$293.1364, found 293.1358.

2,5-二(4'-丁基苯基)噻吩(2c): $245 \mathrm{mg}$, 淡黄色固体, m.p.130 131 ${ }^{\circ} \mathrm{C}$ (lit. $\left.{ }^{[5 \mathrm{c}]} 132 \sim 134{ }^{\circ} \mathrm{C}\right) ;{ }^{1} \mathrm{H}$ NMR $\left(\mathrm{CDCl}_{3}\right.$, $400 \mathrm{MHz}) \delta: 0.93\left(\mathrm{t}, J=7.6 \mathrm{~Hz}, 6 \mathrm{H}, \mathrm{CH}_{3}\right), 1.34 \sim 1.38(\mathrm{~m}$, $\left.4 \mathrm{H}, \mathrm{CH}_{2}\right), 1.59 \sim 1.63\left(\mathrm{~m}, 4 \mathrm{H}, \mathrm{CH}_{2}\right), 2.63$ (q, $J=7.6 \mathrm{~Hz}$, $\left.4 \mathrm{H}, \mathrm{CH}_{2}\right), 7.19(\mathrm{~d}, J=8.0 \mathrm{~Hz}, 4 \mathrm{H}, \mathrm{PhH}), 7.23$ (s, 2H, ArH), $7.53(\mathrm{~d}, J=8.4 \mathrm{~Hz}, 4 \mathrm{H}, \mathrm{PhH}) ;{ }^{13} \mathrm{C}$ NMR $\left(\mathrm{CDCl}_{3}, 100\right.$ $\mathrm{MHz}) \delta: 13.98,22.37,33.56,35.37,123.43,125.51$, 128.95, 131.87, 142.37, 143.29; HRMS (ESI) calcd for $\mathrm{C}_{24} \mathrm{H}_{29} \mathrm{~S}[\mathrm{M}+\mathrm{H}]^{+}$349.1990, found 349.2011.

2,5-二(4'-乙氧基苯基)噻吩(2d): $176 \mathrm{mg}$, 白色固体, m.p. $216 \sim 218{ }^{\circ} \mathrm{C} ;{ }^{1} \mathrm{H}$ NMR $\left(\mathrm{CDCl}_{3}, 400 \mathrm{MHz}\right) \delta: 1.43$ (t, $\left.J=7.2 \mathrm{~Hz}, 6 \mathrm{H}, \mathrm{CH}_{3}\right), 4.06\left(\mathrm{q}, J=7.2 \mathrm{~Hz}, 4 \mathrm{H}, \mathrm{CH}_{2}\right), 6.91$ (d, $J=8.8 \mathrm{~Hz}, 4 \mathrm{H}, \mathrm{PhH}), 7.14$ (s, 2H, ArH), 7.53 (d, $J=$ $8.8 \mathrm{~Hz}, 4 \mathrm{H}, \mathrm{PhH}) ;{ }^{13} \mathrm{C}$ NMR $\left(\mathrm{CDCl}_{3}, 100 \mathrm{MHz}\right) \delta: 14.9$, $63.6,114.9,122.8,126.8,127.2,142.6,158.6$; HRMS (ESI) calcd for $\mathrm{C}_{20} \mathrm{H}_{21} \mathrm{O}_{2} \mathrm{~S}[\mathrm{M}+\mathrm{H}]^{+}$325.1262, found 325.1254 .

2,5-二(4'-甲基苯基)噻吩(2e $)^{[4 \mathrm{p}]}$ : $227 \mathrm{mg}$, 白色固体, m.p. $157 \sim 159{ }^{\circ} \mathrm{C}$ (lit. $\left.{ }^{[8]} 161 \sim 164{ }^{\circ} \mathrm{C}\right) ;{ }^{1} \mathrm{H} \mathrm{NMR}\left(\mathrm{CDCl}_{3}\right.$, $400 \mathrm{MHz}) \delta$ : 2.37(s, 6H, $\left.\mathrm{CH}_{3}\right), 7.19(\mathrm{~d}, J=7.6 \mathrm{~Hz}, 4 \mathrm{H}$, $\mathrm{PhH}), 7.23$ (s, 2H, ArH), 7.52 (d, $J=8.0 \mathrm{~Hz}, 4 \mathrm{H}, \mathrm{PhH}) ;{ }^{13} \mathrm{C}$ NMR $\left(\mathrm{CDCl}_{3}, 100 \mathrm{MHz}\right) \delta$ : 21.2, 123.4, 125.5, 129.6,
131.7, 137.3, 143.3; HRMS (ESI) calcd for $\mathrm{C}_{18} \mathrm{H}_{17} \mathrm{~S}[\mathrm{M}+$ $\mathrm{H}]^{+}$265.1051, found 265.1049 .

2,5-二(4'-甲氧基苯基)噻吩 (2f ${ }^{[\mathrm{5c}]}: 164 \mathrm{mg}$, 白色固 体, m.p. $192 \sim 194{ }^{\circ} \mathrm{C}$ (lit. ${ }^{[5 \mathrm{cc}} 216 \sim 217{ }^{\circ} \mathrm{C}$ ); ${ }^{1} \mathrm{H}$ NMR $\left(\mathrm{CDCl}_{3}, 400 \mathrm{MHz}\right) \delta: 3.84\left(\mathrm{~s}, 6 \mathrm{H}, \mathrm{CH}_{3}\right), 6.92(\mathrm{~d}, J=8.8$ $\mathrm{Hz}, 4 \mathrm{H}, \mathrm{PhH}), 7.25$ (s, 2H, ArH), 7.54 (d, $J=8.4 \mathrm{~Hz}, 4 \mathrm{H}$, $\mathrm{PhH}) ;{ }^{13} \mathrm{C}$ NMR $\left(\mathrm{CDCl}_{3}, 100 \mathrm{MHz}\right) \delta: 55.39,114.31$, 122.90, 126.84, 134.06, 142.60,159.12; HRMS (ESI) calcd for $\mathrm{C}_{18} \mathrm{H}_{17} \mathrm{O}_{2} \mathrm{~S}[\mathrm{M}+\mathrm{H}]^{+}$297.0949, found 297.0947.

2,5-二(3'-氨基苯基)噻吩 $(\mathbf{2 g})^{[7]}: 198 \mathrm{mg}$, 白色固体, m.p. 202 204 ${ }^{\circ} \mathrm{C}\left(\right.$ lit. $\left.{ }^{[7]} 202{ }^{\circ} \mathrm{C}\right) ;{ }^{1} \mathrm{H}$ NMR $\left(\mathrm{CDCl}_{3}, 400\right.$ MHz) $\delta: 3.72\left(\mathrm{~s}, 4 \mathrm{H}, \mathrm{NH}_{2}\right), 6.60 \sim 6.62(\mathrm{~m}, 2 \mathrm{H}, \mathrm{PhH}), 6.94$ (m, 2H, PhH), 7.02 7.05 (m, 2H, PhH), 7.14 7.18 (m, $2 \mathrm{H}, \mathrm{ArH}), 7.22(\mathrm{~s}, 2 \mathrm{H}, \mathrm{PhH}) ;{ }^{13} \mathrm{C} \mathrm{NMR}\left(\mathrm{CDCl}_{3}, 100 \mathrm{MHz}\right)$ $\delta: 112.2,114.4,116.2,123.8,129.6,135.7,143.6,146.6$; HRMS (ESI) calcd for $\mathrm{C}_{16} \mathrm{H}_{15} \mathrm{~N}_{2} \mathrm{~S}[\mathrm{M}+\mathrm{H}]^{+}$267.0956, found 267.0953 .

2,5-二(2'-甲基苯基)噻吩 $(\mathbf{2 h})^{[7]}: 190 \mathrm{mg}$, 无色液体. ${ }^{1} \mathrm{H}$ NMR $\left(\mathrm{CDCl}_{3}, 400 \mathrm{MHz}\right) \delta: 2.50\left(\mathrm{~s}, 6 \mathrm{H}, \mathrm{CH}_{3}\right), 7.06$ (s, 2H, ArH), 7.24 7.28 (m, 6H, PhH), 7.47 (m, 2H, PhH); ${ }^{13} \mathrm{C} \mathrm{NMR}\left(\mathrm{CDCl}_{3}, 100 \mathrm{MHz}\right) \delta: 21.3,126.0,126.5,127.8$, 130.3, 130.7, 134.1, 136.0, 142.9; HRMS (ESI) calcd for $\mathrm{C}_{18} \mathrm{H}_{17} \mathrm{~S}[\mathrm{M}+\mathrm{H}]^{+}$265.1051, found 265.1047.

2,5-二(2'-吡啶基)噻吩 $(2 \mathbf{i})^{[5 \mathrm{c}]}: 167 \mathrm{mg}$, 淡黄色固体, m.p. $\quad 154 \sim 157 \quad{ }^{\circ} \mathrm{C} \quad$ (lit. ${ }^{[\mathrm{cc}]} 157 \sim 159{ }^{\circ} \mathrm{C} ;{ }^{1} \mathrm{H}$ NMR $\left(\mathrm{CDCl}_{3}, 400 \mathrm{MHz}\right) \delta: 7.16 \sim 7.20(\mathrm{~m}, 2 \mathrm{H}, \mathrm{ArH}), 7.65 \sim$ $7.75(\mathrm{~m}, 6 \mathrm{H}, \mathrm{PhH}), 8.60(\mathrm{~d}, J=4.8 \mathrm{~Hz}, 2 \mathrm{H}, \mathrm{PhH}) ;{ }^{13} \mathrm{C}$ NMR $\left(\mathrm{CDCl}_{3}, 100 \mathrm{MHz}\right) \delta: 112.2,114.4,116.2,123.8$, 129.6, 135.4, 146.6. HRMS (ESI) calcd for $\mathrm{C}_{14} \mathrm{H}_{11} \mathrm{~N}_{2} \mathrm{~S}$ $[\mathrm{M}+\mathrm{H}]^{+}$239.0643, found 239.0641.

辅助材料(Supporting Information) 合成产物的 ${ }^{1} \mathrm{H}$ $\mathrm{NMR}$ 和 ${ }^{13} \mathrm{C}$ NMR 谱图. 这些材料可以免费从本刊网站 (http://sioc-journal.cn/)上下载.

\section{Referenes}

[1] For selected review, see: (a) Wu, X. F.; Neumann, H.; Beller, M. Chem. Rev. 2013, 113, 1 .

(b) Zeni, G.; Larock, R. C. Chem. Rev. 2006, 106, 4644.

(c) Lipshutz, B. H. Chem. Rev. 1986, 86, 795.

(d) Lu, H.; Liu, G. T. Planta Med. 1992, 58, 311.

(e) Navarro, E.; Alonso, S. J.; Trujillo, J.; Jorge, E.; Pérez, C. J. Nat. Prod. 2001, 64, 134.

(f) Cacchi, S.; Fabrizi, G.; Goggiamani, A. Org. Biomol. Chem. 2011, 9, 641 .

(g) Flynn, B. L.; Hamel, E.; Jung, M. K. J. Med. Chem. 2002, 45, 2670.

(h) Palkowitz, A. D.; Glasebrook, A. L.; Thrasher, K. J.; Hauser, K. L.; Short, L. L.; Philips, D. L.; Muehl, B. S.; Sato, M.; Shetler, P. K.; Cullinan, G. J.; Pell, T. R.; Bryant, H. U. J. Med. Chem. 1997, 
$40,1407$.

(i) Tsuji, H.; Cantagrel, G.; Ueda, Y.; Chen, T.; Wan, L. J.; Nakamura, E. Chem. Asian J. 2013, 8, 2377.

[2] Joule, J. A.; Mills, K. Heterocyclic Chemistry, Trans. by You, Y.-C.; Gao, D.-B. Science Press, Beijing, 2004, p. 324 (in Chinese).

(J. A. 焦耳, K. 米尔斯, 杂环化学, 由业诚, 方大彬译, 科学出 版社，北京, 2004, p. 324.)

[3] (a) Snégaroff, K.; Komagawa, S.; Chevallier, F.; Gros, P. C.; Golhen, S.; Roisnel, T.; Uchiyama, M.; Mongin, F. Chem.-Eur. J. 2010, 16, 8191 .

(b) Kel'in, A. V.; Gevorgyan, V. J. Org. Chem. 2002, 67, 95.

(c) Rao, H. S. P.; Jothilingam, S. J. Org. Chem. 2003, 68, 5392

(d) Aponick, A.; Li, C. Y.; Malinge, J.; Marques, E. F. Org. Lett. 2009, 11, 4624 .

(e) Egi, M.; Azechi, K.; Akai, S. Org. Lett. 2009, 11, 5002.

(f) Dheur, J.; Sauthier, M.; Castanet, Y.; Mortreux, A. Adv. Synth. Catal. 2010, 352, 557.

[4] (a) Sun, H.; Wu, X.; Hua, R. Tetrahedron Lett. 2011, 52, 4408.

(b) Singha, R.; Nandi, S.; Ray, J. K. Tetrahedron Lett. 2012, 53, 6531.

(c) Pridmore, S. J.; Slatford, P. A.; Williams, J. M. J. Tetrahedron Lett. 2007, 48, 5111.

(d) Kramer, S.; Madsen, J. L. H.; Rottl€ander, M.; Skrydstrup, T. Org. Lett. 2010, 12, 2758.

(e) Nun, P.; Dupuy, S.; Gaillard, S.; Poater, A.; Cavallod, L.; Nolan, S. P. Catal. Sci. Technol. 2011, 1, 58.

(f) Jiang, H.; Zeng, W.; Li, Y.; Wu, W.; Huang, L.; Fu, W. J. Org. Chem. 2012, 77, 5179. (g) Zheng, Q.; Hua, R.; Yin, T. Curr. Org. Synth. 2013, 10, 161.

(h) Beny, J.-P.; Dhawan, S. N.; Kagan, J.; Sundlass, S. J. Org. Chem. 1982, 47, 2201.

(i) Pridmore, S. J.; Slatford, P. A.; Daniel, A.; Whittlesey, M. K.; Williams, J. M. J. Tetrahedron Lett. 2007, 48, 5115

(j) Lavallo, V.; Frey, G. D.; Donnadieu, B.; Soleilhavoup, M.; Bertrand, G. Angew. Chem., Int. Ed. 2008, 47, 5224.

(k) Zheng, Q.; Hua, R. Tetrahedron Lett. 2010, 51, 4512.

(1) Mandadapu, A. K.; Sharma, S. K.; Gupta, S.; Krishna, D. G. V.; Kundu, B. Org. Lett. 2011, 13, 3162.

(m) Mandadapu, A. K.; Dathi, M. D.; Arigela, R. K.; Kundu, B. Tetrahedron 2012, 68, 8207.

(n)Wang, L.; Yu, X.; Feng, X.; Bao, M. Org. Lett. 2012, 14, 2418.

(o) Zheng, Q.; Hua, R.; Yin, T. Curr. Org. Synth. 2013, 10, 161.

(p) Jiang, H.; Zeng, W.; Li, Y.; Wu, W.; Huang, L.; Fu, W. J. Org. Chem. 2012, 77, 5179.

[5] (a) Zheng, Q.; Hua, R.; Jiang, J.; Zhang, L. Tetrahedron 2014, 70, 8252 .

(b) Zhang, G.; Yi, H.; Chen, H.; Bian, C.; Liu, C.; Lei, A. Org. Lett. 2014, 16, 6156.

(c) Tang, J.; Zhao, X. RSC Adv. 2012, 2, 5488.

[6] (a) Li, D.; Yin, K.; Li, J.; Jia, X. Tetrahedron Lett. 2008, 49, 5918. (b) Li, L.; Wang, J.; Zhang, G.; Liu, Q. Tetrahedron Lett. 2009, 50, 4033.

[7] Urselmann, D.; Antovic, D.; Müller T. Beilstein J. Org. Chem. 2011, 7, 1499

[8] Irudayanathan, F. M.; Raja, G. C. E.; Lee, S. Tetrahedron 2015, 71, 4418.

(Li, L.; Fan, Y.) 